\title{
Increasing Prevalence of Non-Clade B HIV-1 Strains in Heterosexual Men and Women, as Monitored by Analysis of Reverse Transcriptase and Protease Sequences
}

\author{
*Claudia Balotta, *Guido Facchi, *Michela Violin, †Sonia Van Dooren, †Alessandro Cozzi-Lepri, \\ $\S$ Federica Forbici, §Ada Bertoli, *Chiara Riva, *Daniela Senese, IIPietro Caramello, \\ **Giuseppe Carnevale, $\dagger \dagger$ Giuliano Rizzardini, $+\neq$ Laura Cremonini, §§Laura Monno, \\ IIIGiovanni Rezza, §Carlo Federico Perno, §Giuseppe Ippolito, *Antonella d'Arminio-Monforte, \\ $\dagger$ Anne-Mieke Vandamme, and *Mauro Moroni, for the ICONA Study Group \\ *Institute of Infectious and Tropical Diseases, “L. Sacco” Hospital, University of Milan, Italy; †Rega Institute and University \\ Hospitals, University of Leuven, Belgium; $\ddagger$ Royal Free and University College Medical School, Royal Free Campus, London, \\ U.K.; \$IRCCS, “L. Spallanzani," Rome, Italy; IIDepartment of Infectious Diseases, “Amedeo di Savoia” Hospital, Turin; \\ **Department of Infectious Diseases, Istituti Ospitalieri di Cremona; ††Department of Infectious Diseases, Busto Arsizio \\ Hospital, Varese; $\$+$ Infectious Diseases Clinic, University of Modena and Reggio, Emilia; $\$$ Infectious Diseases Clinic, \\ University of Bari; and IIIIstituto Superiore di Sanità, Rome, Italy
}

\footnotetext{
Address correspondence and reprint requests to Claudia Balotta, Institute of Infectious and Tropical Diseases, "L. Sacco" Hospital, University of Milan, Via G. B. Grassi, 74, 20157 Milano, Italy; e-mail: claudia.balotta@unimi.it

Manuscript received January 2, 2001; accepted May 3, 2001.
}

\begin{abstract}
Objective: We evaluated the prevalence of HIV-1 non-clade B over time in a formerly clade B-restricted area. Protease and reverse transcriptase regions of the pol gene were used for phylogenetic and recombination analysis and for clade assignment to HIV-1 A-D, F-H, J, and K strains of the M group.

Methods: The pol gene of $349 \mathrm{HIV}-1$ patients belonging to the Italian Cohort Naive for Antiretrovirals (ICONA) were genotypically analyzed to study the prevalence of viral-associated resistance mutations. All HIV-1 pol sequences and $32 \mathrm{HIV}$ reference strains were analyzed, including the reference strains for the major HIV-1 subtypes. The non-clade B sequences according to the HIV-1 Subtyping Tool program were further studied by a bootscan analysis (SimPlot) to investigate the likelihood of combination between subtypes. The proportions of patients carrying non-clade B virus before and after 1997 were female gender were significantly associated with the presence of non-clade B subtypes ( $p=.001$ and .005 , respectively). Non-clade B HIV-1 was harbored by $14.5 \%$ of the heterosexuals who were found to be HIV-1 positive after 1997, 60\% of whom were women. Bootscan analysis identified four strains as F, two as A, one as C, one as G, and $11(57.9 \%)$ as non-clade B recombinant subtypes.

Conclusion: Detection of HIV-1 subtypes and intersubtype recombinants in a previously clade B-homogeneous area indicates that the HIV-1 epidemic is evolving in Italy and that heterosexuals and women are at increased risk of infection with nonclade B HIV-1 subtypes. Sequences inferred from the pol gene yield to establish the subtype of circulating HIV-1 strains. As a consequence, genotyping of pol gene for testing resistance to antiretrovirals warrants concomitant surveillance of non-clade B subtypes.

Key Words: HIV-1 subtypes-Circulating HIV-1 recombinant forms-HIV-1 pol sequences-Italian Cohort Naive for Antiretrovirals.
\end{abstract}


types of group $\mathrm{M}(\mathrm{A}-\mathrm{D}, \mathrm{F}-\mathrm{H}, \mathrm{J}$, and $\mathrm{K}$ ) circulate to varying extents in populations around the globe (3). Mosaic genomes of HIV-1 have recently been recognized and called circulating recombinant forms (CRFs), six of which are currently identified as $\mathrm{AE}, \mathrm{AG}, \mathrm{AB}, \mathrm{DF}$, AGHK, and AGJK on the basis of full-length viral sequences $(4,5)$. CRFs show a mosaic genomic structure resulting from intersubtype recombinations, and their presence probably reflects multiple infections in humans. These viruses have become geographically widespread and are likely to play an important role in the HIV pandemic (3).

Although viruses belonging to the HIV-1 B clade are still predominant in Europe, the United States, and Australia, an increasing prevalence of non-clade B subtypes has been reported by several surveys in previously homogeneous clade-B countries (6-12). Accurate methods are therefore urgently needed to detect HIV-1 nucleic acids and monitor patients infected with non-clade B strains.

In addition to serologic procedures, HIV-1 subtypes are currently classified by the amplification and sequencing of portions of env and gag structural genes and inferring their phylogenetic relationships (13). Over the last few years, attempts to monitor the distribution of distinct clades have been paralleled by efforts to test drug-resistance by molecular methods based on polymerase chain reaction (PCR) and automated sequence technology applied to HIV-1 nucleic acids (14). The genotyping of HIV-1 pol reverse transcriptase (RT) and protease (PR) regions generates long stretches of nucleotides (approximately 1,200 base pairs [bp]). These sequences can be used to define clade clustering with the aid of the specific HIV-1 subtyping tool available on the Word Wide Web (http://hiv-web.lanl.gov/). The intersubtype recombination of non-clade B strains may also be assessed using dedicated software (SimPlot, Baltimore, MD, U.S.A.) (15).

In this study, we evaluated the presence of non-clade B HIV-1 subtypes in a formerly clade B-restricted area using pol sequences generated for drug-resistance testing. This analysis allowed monitoring of the prevalence of non-clade B subtypes by risk categories and genders, which may change with the evolution of the HIV-1 epidemic in European countries.

\section{METHODS}

All the patients gave their informed consent to participate in the study. The study was conducted in accordance with Italian Ministry of Health Guidelines.

The participants were a subset of the Italian observational cohort of HIV-1-infected individuals naive for antiretrovirals at the time of en- rollment (I.CO.N.A.), whose demographic, immunologic, virologic, clinical, and therapeutic parameters are registered in a comprehensive database (16).

The HIV-1 RT and PR regions of the pol gene of 349 I.CO.N.A. patients (68 of whom had seroconverted in the preceding 12 months) were genotypically analyzed to study the prevalence of antiretroviralassociated resistance mutations and investigate their role in predicting virologic failure during highly active antiretroviral therapy (HAART). The date of the first HIV-1 antibody determination was used as a surrogate of the time of non-clade B subtype penetration in Italy for patients with established infection.

Plasma HIV-1 RNA levels were measured using the following assays: Roche Amplicor (HIV MonitorTM, Roche Diagnostics Corporation, Indianapolis, IN, U.S.A.) in $32.6 \%$ of patients, nucleic acid sequence-based amplification (NASBA) (NucliSens, Organon Teknika Corporation, Durham, NC, U.S.A.) in $15.2 \%$ patients and branched DNA (Quantiplex, Chiron Corporation, Emeryville, CA, U.S.A.) in $52.2 \%$ patients. CD4 T-cell counts were performed by standard cytofluorometric techniques using Coulter (Coulter Electronics, Inc., Miami Lakes, FL, U.S.A.) or Ortho systems (Ortho Diagnostic Systems, Raritan, NJ, U.S.A.). Stage was assigned according to the 1993 U.S. Centers for Disease Control and Prevention (CDC) classification (17).

HIV-1 RNA was extracted, reverse transcripted, amplified, and sequenced on patient plasma samples using version 1.0 of an Applied Biosystems kit (HIV Genotyping System, Applied Biosystems, Foster City, CA, U.S.A.) following the manufacturer's recommendations. The cDNA obtained by reverse-transcription with random hexamers was amplified using sequence-specific primers. Cycle-sequencing dideoxy chain termination chemistry with seven different sequence-specific primers was used to obtain a 1,260-kb sequence on an ABI-377 automated sequencer (Applied Biosystems). Dedicated software (HIV Genotyping System SoftwareTM, Applied Biosystems, Foster City, CA, U.S.A.) that assembles a consensus sequence and compares it with a NL4-3 reference strain was used to analyze the data.

The 349 sequences were edited, assembled, and trimmed to equivalent length $(1,229 \mathrm{bp})$ using the Sequence Navigator program (Applied Biosystems). Sequences were aligned with the PR and RT sequences of 32 HIV-1 group M A-J reference strains available in the Los Alamos database (1999 subtype reference alignment: http://hiv-web.lanl.gov/. accessed July, 2000).

The sequence of each patient was first analysed using the HIV-1 subtyping tool (available at the http://www.ncbi.nlm.nih. gov/retroviruses/subtype/subtype.html), which allows rapid subtype evaluation, and then submitted to a bootscan analysis (SimPlot v2.5, available at http://www.med.jhu.edu/deptmed/sray/download) to analyze the possibility of subtype recombinations (15). We performed a neighbor-joining boot scan by comparing the query sequence with reference strains using a sliding window of 400 nucleotides (nt) and moving forward with steps of $20 \mathrm{nt}$. The subtype assignment of the non-clade B strains was confirmed by a bootstrapped phylogenetic analysis using SEQBOOT with 1000 replicates, followed by the DNAdist, Neighbor, and Consense programs (18). For the recombinant strains, stretches of the A, B, F, and G subtypes were recognised by boot-scanning but not further analyzed for their possible recombination patterns. The sequences of the non-clade B subtypes were submitted to Genbank with the assigned accession numbers of AF295281 to 295299.

Standard nonparametric methods (Wilcoxon signed-rank test) were used to compare the median age, HIV-1 RNA levels, and CD4 counts in the patients with B and non-clade B subtypes. The proportions of study subjects in these two groups were compared using Fisher exact and $\chi^{2}$ tests. The crude and Mantel-Haenszel adjusted odds ratios (OR) of having a non-clade B subtype were also calculated. The 
presence of interactions between two factors was verified by using the Breslow-Day test for the homogeneity of OR.

\section{RESULTS}

Table 1 shows the demographic, clinical, virologic, and immunologic characteristics of the patients with B and non-clade B subtypes. The overall prevalence of non-clade B subtypes in our study population was 5.4\% (19 cases).

The number of study subjects harboring non-clade B HIV-1 subtypes increased over time from 3 of 158 patients $(1.9 \%)$ who had had a first positive HIV-1 antibody test result before 1997 to 16 of 191 (8.4\%) of those with a first positive antibody determination after 1997 $(p=.008)$. The median age of the patients with nonclade B variants was 42 years (range, 34-48) compared with 34 years (range, 30-38) in those with B subtypes $(p=.003)$. Eight out of 81 female (9.9\%) and 11 of 268 male patients $(4.1 \%)$ harbored a non-clade B HIV-1 sub- type $(p=.054)$. Of the 6 patients with African citizenship, 5 harbored non-clade B HIV-1 subtypes. The proportion of study subjects with non-clade B HIV-1 was significantly higher in Africans (83.3\%) than in whites $(4.2 \%)$ or other races $(0 \%)(p<.0001)$.

The modalities of HIV-1 transmission in patients with non-clade B subtypes were heterosexual sex in 15 cases (78.9\%), homosexual sex in $3(15.8 \%)$, and intravenous drug use in $1(5.3 \%)$. The proportion of non-clade B HIV-1 subtypes was significantly higher in those subjects at risk through heterosexual contact (15 of 107, $14.0 \%$ ) than in homosexuals ( 3 of $92,3.3 \%$ ) or intravenous drug users (IVDUs) ( 1 of $124,0.8 \%)(p=.001)$.

No difference was present in the proportion of clade B and non-clade B strains between the patients with different degrees of disease severity according to the 1993 CDC classification. The median HIV-1 RNA was 96,950 copies $/ \mathrm{ml}$ and the median CD4 count 245 cells $/ \mu 1$ in the individuals harboring non-clade B subtypes; the corresponding figures for those with clade B subtypes were

TABLE 1. Demographic, clinic, immunologic, and virologic characteristics of 349 Italian Cohort Naive for Antiretrovirals study patients subjected to subtype analysis of protease and reverse transcriptase regions of pol gene

\begin{tabular}{|c|c|c|c|c|}
\hline & $\begin{array}{l}\text { B-clade } \\
\text { subtype }\end{array}$ & $\begin{array}{l}\text { Non-clade B } \\
\text { subtype }\end{array}$ & $\begin{array}{c}\text { All } \\
\text { patients }\end{array}$ & $p$ value $^{a}$ \\
\hline \multicolumn{5}{|l|}{ 1st HIV-1 antibody test (year: $\%, n$ ) } \\
\hline$<1997$ & $98.1(155)$ & $1.9(3)$ & $45.3(158)$ & \multirow[t]{2}{*}{.008} \\
\hline$\geq 1997$ & $91.6(175)$ & $8.4(16)$ & $54.7(191)$ & \\
\hline Age (yr), median & 34 & 42 & 34 & .003 \\
\hline (25\%-75\% quartiles) & $(30-38)$ & $(34-48)$ & $(30-48)$ & \\
\hline \multicolumn{5}{|l|}{ Gender $\%,(n)$} \\
\hline Male & $95.9(257)$ & $4.1(11)$ & $76.8(268)$ & \multirow[t]{2}{*}{.054} \\
\hline Female & $90.1(73)$ & $9.9(8)$ & $23.2(81)$ & \\
\hline \multicolumn{5}{|l|}{ Race $\%,(n)$} \\
\hline White $^{b}$ & $95.8(317)$ & $4.2(14)$ & $94.9(331)$ & \multirow[t]{3}{*}{$<.0001$} \\
\hline African & $16.7(1)$ & $83.3(5)$ & $1.7(6)$ & \\
\hline Other ${ }^{c}$ & $100.0(12)$ & $0.0(0)$ & $3.4(12)$ & \\
\hline \multicolumn{5}{|l|}{ Risk factor $\%,(n)$} \\
\hline Heterosexual sex & $86.0(92)$ & $14.0(15)$ & 30.7 (107) & \multirow[t]{4}{*}{.001} \\
\hline Homosexual sex & $96.7(89)$ & $3.3(3)$ & $26.4(92)$ & \\
\hline Injection use & $99.2(123)$ & $0.8(1)$ & $35.5(124)$ & \\
\hline Other ${ }^{d}$ & $100.0(26)$ & $0.0(0)$ & $7.4(26)$ & \\
\hline \multicolumn{5}{|l|}{1993 CDC stage $\%,(n)$} \\
\hline A & $95.3(223)$ & $4.7(11)$ & $68.8(234)$ & \multirow[t]{3}{*}{.25} \\
\hline $\mathrm{B}$ & $95.8(46)$ & $4.2(2)$ & $14.1(48)$ & \\
\hline $\mathrm{C}$ & $89.7(52)$ & $10.3(6)$ & $17.1(58)$ & \\
\hline \multirow{2}{*}{$\begin{array}{l}\text { CD4 count (cells/ } \mu 1) \text { median } \\
(25 \%-75 \% \text { quartiles })\end{array}$} & 339 & 245 & 338 & \multirow[t]{2}{*}{.35} \\
\hline & $(100-512)$ & $(87-419)$ & $(109-511)$ & \\
\hline \multirow{2}{*}{$\begin{array}{l}\text { HIV-1 RNA (copies/ml) median } \\
\quad(25 \%-75 \% \text { quartiles })\end{array}$} & 63,064 & 96,950 & 69,000 & \multirow[t]{2}{*}{.83} \\
\hline & $(22,000-230,000)$ & $(28,500-227,455)$ & $(22,000-230,000)$ & \\
\hline Total patients $\%,(n)$ & $94.6(330)$ & $5.4(19)$ & $100.0(349)$ & - \\
\hline
\end{tabular}


63,066 copies $/ \mathrm{ml}$ and 339 cells $/ \mu l$, respectively. These differences were not significant. These data are shown in Table 1.

We then investigated which of the factors univariately associated with a higher proportion of non-clade B subtype was an independent predictor of this outcome and, in particular, whether the increase in the proportion of non-clade B subtypes occurring after 1997 could be explained by the increase in the proportion of patients who acquired HIV through heterosexual contact or those who were of African origin. The OR of having a non-clade B subtype after 1997 differed among those patients infected through heterosexual contacts and those infected through other routes: the increase in non-clade B subtypes after 1997 among heterosexuals (from 0 to 15 cases, $19.5 \%$ ) and the decrease in the patients infected through other routes showed that heterosexual exposure is an effect-modifier rather than a confounding factor (OR, 0.37, Breslow-Day test for homogeneity of OR; $p=.007)$. Because no African patient tested seropositive before 1997, it was not possible to calculate the adjusted OR for African race but, after adjusting for white race, the OR of having non-clade B infection after 1997 was $4.23(p=.02)$. The effect of age also resulted to be independent of the year of testing (crude OR, 2.00 for 10 years older, $p=.002$; OR, 1.67, $p=.07$, after adjusting for the other factors in Table 1). Some of the analyses were restricted to whites. Table 2 shows gender and modality of infection in 331 whites grouped according to the year of first positive HIV-1 antibody test result. Three patients (2.0\%) had non-clade B before 1997 and $11(6.2 \%)$ after $1997\left(\chi^{2} p=.06\right)$. The three non-clade B strains identified before 1997 were only found among men. Those identified after 1997 were found in 5 men and 6 women. The proportion of women with a nonclade B subtype increased from $0.0 \%$ to $17.7 \%$ even though the male:female ratio increased from 2.6 to 4.2 during the period of observation. The increase in the proportion of patients with non-clade B strains therefore cannot be explained on the basis of the higher proportion of women enrolled in recent years, but the proportion of patients harboring non-clade $\mathrm{B}$ viruses significantly increased over time among women but not among men (Breslow-Day test for homogeneity of OR, $p=.04$ ).

Regarding the distribution of patients among risk categories, heterosexuals and homosexuals increased from $19 \%$ to $38.8 \%$ and $13.7 \%$ to $34.8 \%$ before and after 1997 , respectively. In contrast, IVDUs were $62.1 \%$ and $16.3 \%$ overtime. Among the white patients, the prevalence of non-clade B subtypes increased from $0.0 \%$ to $14.5 \%$ in heterosexuals, decreased in homosexuals and remained at a similar low level in IVDUs. The non-clade B strains identified before $1997(n=3)$ were found in 2 homosexuals and 1 male IVDU, but the 11 non-clade B subtypes identified after 1997 were found in 1 homosexual, 4 heterosexual men, and 6 heterosexual women.

TABLE 2. Gender and risk factors in 331 white patients according to the year of 1 st positive HIV-1 determination

\begin{tabular}{|c|c|c|c|c|c|c|c|c|}
\hline & \multicolumn{3}{|c|}{$<1997$} & \multicolumn{3}{|c|}{$\geq 1997$} & \multirow[b]{2}{*}{$\begin{array}{c}\text { Total } \\
\% \text { of column }\end{array}$} & \multirow[b]{2}{*}{$p$ value $^{a}$} \\
\hline & $\begin{array}{c}\text { Total } \\
\% \text { of column }\end{array}$ & $\begin{array}{l}\text { B clade } \\
\% \text { of row }\end{array}$ & $\begin{array}{l}\text { Non-B clade } \\
\% \text { of row }\end{array}$ & $\begin{array}{c}\text { Total } \\
\% \text { of column }\end{array}$ & $\begin{array}{l}\text { B clade } \\
\% \text { of row }\end{array}$ & $\begin{array}{c}\text { Non-B clade } \\
\% \text { of row }\end{array}$ & & \\
\hline \multicolumn{9}{|l|}{ Gender } \\
\hline \multirow[t]{2}{*}{ Men } & 111 & 108 & 3 & 144 & 139 & 5 & 255 & .005 \\
\hline & 72.5 & 97.3 & 2.7 & 80.9 & 96.5 & 3.5 & 77.0 & \\
\hline \multirow[t]{2}{*}{ Women } & 42 & 42 & 0 & 34 & 28 & 6 & 76 & \\
\hline & 27.5 & 100.0 & 0.0 & 19.1 & 82.3 & 17.7 & 23.0 & \\
\hline \multicolumn{9}{|l|}{ Risk Factors } \\
\hline \multirow[t]{2}{*}{ Heterosexuals $^{b}$} & 29 & 29 & 0 & 69 & 59 & 10 & 98 & \\
\hline & 19.0 & 100.0 & 0.0 & 38.8 & 85.5 & 14.5 & 29.6 & \\
\hline \multirow[t]{2}{*}{ Men } & 12 & 12 & 0 & 41 & 37 & 4 & 53 & \\
\hline & 41.4 & 41.4 & 0.0 & 59.4 & 62.7 & 40.0 & 16.0 & \\
\hline \multirow[t]{2}{*}{ Women } & 17 & 17 & 0 & 28 & 22 & 6 & 45 & \\
\hline & 58.6 & 58.6 & 0.0 & 40.6 & 37.3 & 60.0 & 13.6 & .001 \\
\hline \multirow[t]{2}{*}{ Homosexuals } & 21 & 19 & 2 & 62 & 61 & 1 & 83 & \\
\hline & 13.7 & 90.5 & 9.5 & 34.8 & 98.4 & 1.6 & 25.1 & \\
\hline \multirow[t]{2}{*}{ Injection drug users } & 95 & 94 & $1^{c}$ & 29 & 29 & 0 & 124 & \\
\hline & 62.1 & 98.9 & 1.1 & 16.3 & 100.0 & 0.0 & 37.5 & \\
\hline \multirow[t]{2}{*}{ Other } & 8 & 8 & 0 & 18 & 18 & 0 & 26 & \\
\hline & 5.2 & 100.0 & 0.0 & 10.1 & 100.0 & - & 7.8 & \\
\hline \multirow[t]{2}{*}{ Total } & 153 & 150 & 3 & 178 & 167 & 11 & 331 & - \\
\hline & 100.0 & 98.0 & 2.0 & 100.0 & 93.8 & 6.2 & 100.0 & \\
\hline
\end{tabular}

${ }^{a}$ The $p$ values ( $\chi^{2}$ test) of the comparisons of patients' gender and risk factor before and after 1997.

${ }^{b}$ The proportion of men and women among heterosexuals are shown as absolute numbers and percentages.

${ }^{c}$ Male patient. 
Among the white patients at risk for heterosexual infection $(n=98)$, the percentage of non-clade B subtypes after 1997 was higher among women (6 of 45, 13.3\%) than men $(4$ of $53,7.5 \%)(p=.001)$. More detailed information was available regarding the modality of infection in 7 of the 10 heterosexuals who seroconverted after 1997: 4 were infected as a result of sex with multiple partners and 3 because their partners had multiple partners.

SimPlot bootscan analysis revealed the presence of 11 of 19 HIV-1 subtypes (57.9\%) with a mosaic structure that resulted from intersubtype recombinations in the pol gene. The remaining eight non-clade B subtypes clustered unambiguously within F1 $(n=4), \mathrm{A}(n=2), \mathrm{C}$ $(n=1)$, and $\mathrm{G}(n=1)$ in the neighbor-joining tree. Bootstrap values for these six strains were above $90 \%$ (data not shown).

Overall, non-clade B subtypes were present in 17 of 281 individuals with established infection (6.0\%) and in 2 of $68(2.9 \%)$ recently infected study subjects, 1 man and 1 woman who seroconverted in 1997. The strains were clade $\mathrm{F}$ in one case and a non-clade $\mathrm{B}$ recombinant subtype in the other. Both had had heterosexual sex as risk factor for HIV-1 infection. Among the patients with established infection, 5 ( 3 men and 2 women) were African heterosexuals who became infected in their country of origin (Nigeria, Ivory Coast, Burkina Faso, Equatorial Guinea, and Ghana), and harbored recombinant HIV-1 subtypes. The seroconverters did not differ from the patients with established infection in terms of gender, race, or HIV-1 exposure, but they were significantly younger (median age 32 versus 34 years; Wilcoxon test, $p=.023$ ).

\section{DISCUSSION}

The detection of A to K HIV-1 subtypes and CRFs is of crucial importance in the epidemiologic tracking of the AIDS pandemic. Despite the waves of immigration from African countries to Europe in the 1990s, the prevalence of non-clade B HIV-1 strains is still low in Italy in comparison with other Mediterranean countries or European nations with various relationships with African countries (19). The prevalence detected in our case-file is comparable with that reported in the United States and Canada (7\% and 4\%) (10), although a recent study of more than 5,000 American HIV-1-infected individuals found a lower prevalence $(0.15 \%)(20)$.

The presence of non-clade B subtypes in an area formerly restricted to clade $\mathrm{B}$ infection indicates that the HIV-1 epidemic is evolving in Europe. A recent survey on samples collected between 1984 and 1997 did not reveal the penetration of non-clade B strains in a limited number of Italian IVDUs (G. Rezza, personal communication). The proportion of IVDUs in the I.CO.N.A population is $35.5 \%$, but only 1 of 124 IVDUs $(0.8 \%)$ harbored a non-clade B subtype. It is possible that this risk category does not closely reflect the outbreak of non-clade B strains as has been suggested in another report (19).

The small number of individuals with known seroconversion dates does not allow us to establish a clear date for the arrival of non-clade B subtypes in Italy, so we used the date of the first HIV-1-positive determination as a surrogate for the duration of infection. The overall prevalence of non-clade B subtypes before and after 1997 increased from $1.9 \%$ to $8.4 \%$. Two of the 68 recent seroconverters $(2.9 \%)$ had non-clade B strains. On the basis of these data, we can conclude that the penetration of non-clade B strains occurred in our country before 1997 and possibly continued to increase slightly. The use of the first date of HIV seropositivity as a surrogate marker of seroconversion also implies that the penetration of non-clade B subtypes may actually have occurred before 1997. Furthermore, the seroconverters were younger than the seroprevalent population and this may also explain why the prevalence of non-clade B variants in these individuals was lower than in the patients found to be HIV positive after 1997.

Importantly, the increase in prevalence observed after 1997 could not be explained by the confounding effect of HIV exposure or race. There was a higher proportion of heterosexuals with non-clade B subtypes in the study population as a whole (15 of 19, 78.9\%) and among the white patients (10 of $14,71.4 \%)$. No non-clade B subtypes were observed in heterosexually infected individuals before 1997, but they were found in this risk category during subsequent years $(19.5 \%$ overall; $14.5 \%$ among whites). This difference was significant (homogeneity of OR; $p=.007)$, thus indicating that heterosexual exposure is not a confounder but an effect-modifier. It is also worth noting that $60 \%$ of the heterosexuals with nonclade B strains were women, although this was not unexpected given the increase in the number of heterosexuals over time and the association between heterosexuals and non-clade B subtypes found in this study. Older age also appeared to be independently associated with the presence of non-clade B strains.

$\mathrm{Pol}$ sequences encompassing about 1,200 bp are useful in tracing epidemiologic trends in HIV-1 populations. Their use gives partial information considering a genome of about 10,000 bp. The subtyping is therefore only confident for this region and does not rule out the possibility that other regions belong to different subtypes. 
SimPlot analysis of recombinations in this gene region may not detect recombination breakpoints beyond the pol gene. Almost $60 \%$ of the non-clade B subtypes had the features of recombinant strains, and further investigations are necessary to define their precise nature. Nevertheless, this finding indicates that recombination events occur at high frequency in circulating HIV-1 strains and may influence the distribution of HIV-1 subtypes worldwide.

In conclusion, the penetration and spread of non-clade $\mathrm{B}$ strains can be monitored by genotyping the PR and RT regions of HIV-1 pol gene and using tools available on the World Wide Web and/or phylogenetic analysis. Our data suggest that there was an increase in the prevalence of non-clade B strains in Italy at least after 1997. From the public health perspective, it is important to evaluate the trends of non-clade B diffusion in areas previously restricted to clade B infection and, particularly, between the genders and in the various risk categories. The recent recommendation to test antiretroviral resistance in some clinical settings may help to monitor the spread of different HIV-1 subtypes on a large scale, and the increasing use of antiviral drug resistance genotyping for clinical purposes may allow the concurrent surveillance of circulating HIV-1 subtypes.

\section{APPENDIX}

Members of the ICONA Study Group in Italy include: Ancona: M. Montroni, G. Scalise, A. Costantini, M. S. Del Prete; Aviano (Pordenone): U. Tirelli, G. Nasti. Bari: G. Pastore, M. L. Perulli; Bergamo: F. Suter, C. Arici; Bologna: F. Chiodo, F. M. Gritti, V. Colangeli, C. Fiorini, L. Guerra; Brescia: G. Carosi, G.P. Cadeo, F. Castelli, C. Minardi, D. Vangi; Busto Arsizio: G. Rizzardini, G. Migliorino; $\mathrm{Ca}$ gliari: P. E. Manconi, P. Piano; Catanzaro: T. Ferraro, L. Cosco; Chieti: E. Pizzigallo, F. Ricci; Como: E. Rinaldi, L. Pusterla; Cremona: G. Carnevale, D. Galloni; Cuggiono: P. Viganò, M. Mena; Ferrara: F. Ghinelli, L. Sighinolfi; Firenze: F. Leoncini, F. Mazzotta, S. Ambu, S. Lo Caputo; Foggia: G. Angarano, B. Grisorio, S. Ferrara; Galatina (LE): P. Grima, P. Tundo; Genova: G. Pagano, N. Piersantelli, A. Alessandrini, R. Piscopo; Grosseto: M. Toti, S. Chigiotti; Latina: F. Soscia, L. Tacconi; Lecco: A. Orani, G. Castaldo; Lucca: A. Scasso, A. Vincenti; Mantova: A. Scalzini, F. Alessi; Milano: M. Moroni, A. Lazzarin, A. Cargnel, C. M. Vigevani, L. Caggese, A. d'Arminio Monforte, S. Melzi, F. Delfanti, R. Novati, S. Merli, C. Pastecchia, B. Carini, B. Adriani, S. Garavaglia, C. Moioli; Modena: R. Esposito, C. Mussini; Napoli: N. Abrescia, A. Chirianni, C. Izzo, M. Piazza, M. De Marco, V. Montesarchio, E. Manzillo, S. Nappa; Palermo: A. Colomba, V. Abbadesse, T. Prestileo, S. Mancuso; Pavia: G. Filice, L. Minoli, F. A. Patruno Savino, R. Maserati; Perugia: S. Pauluzzi, F. Baldelli; Pesaro: E. Petrelli, A. Cioppi; Piacenza: F. Alberici, M. Sisti; Pisa: F. Menichetti, A. Smorfa; Potenza: C. De Stefano, A. La Gala; Ravenna: T. Zauli, G. Ballardini; Reggio Emilia: L. Bonazzi, M.A. Ursitti; Rimini: R. Ciammarughi, P. Ortolani; Roma: L. Ortona, F. Dianzani, G. Ippolito, A. Antinori, G. Antonucci, S. D'Elia, P. Narciso, N. Petrosillo, V. Vullo, A. De Luca, L. Del Forno, M. Zaccarelli, P. De
Longis, M. Ciardi, G. D’Offizi, P. Moto, M. Lichtner, M.R. Capobianchi, E. Girardi, P. Pezzotti, G. Rezza; Sassari: M. S. Mura, M. Mannazzu; Torino: P. Caramello, A. Sinicco, M. L. Soranzo, S. Quaglia, M. Sciandra, B. Salassa; Varese: L. Gennero, P. A. Grossi, C. Basilico; Verbania: A. Poggio, G. Bottari; Venezia: E. Raise, S. Pasquinuci; Vicenza: F. De Lalla, G. Tositti; and Taranto: F. Resta, A. Chimienti.

The study was represented in London, England, by A. Cozzi Lepri and A.N. Phillips.

Acknowledgments: This work was financially supported by grants from Istituto Superiore di Sanità, Il Programma Nazionale di Ricerca sull'AIDS-1998 20B.01 to Balotta, 30B.54 and 40B.68 to Moroni, and by an unrestricted educational grant received from Glaxo-Wellcome.

\section{REFERENCES}

1. Expert Group of the Joint United Nations Programme on HIV/AIDS: Implications of HIV variability for transmission: scientific and policy issues. AIDS 1997;1:1-15.

2. European Commission (DG XII JDJUNPoHA) and the Joint United Nations Program on HIV/AIDS: HIV-subtypes: implication for epidemiology, pathogenicity vaccines, and diagnostics. AIDS 1997;11:S17-36.

3. Robertson DL, Anderson JP, Bradac JA, et al. HIV-1 nomenclature proposal. Science 2000, 288:55-6.

4. Carr JK, Foley BT, Leitner T, et al. Reference sequences representing the principle genetic diversity of HIV-1 in the Pandemic. In Human retroviruses and AIDS compendium: a compilation and analysis of nucleic acid and amino acid sequences. Los Alamos, NM:1998.

5. The Circulating Recombinant Forms (CRFs): HIV Sequence Database. Available at http://hiv-web.lanl.gov/. Accessed June 8, 2000.

6. Brodine SK, Mascola JR, Weiss PJ, et al. Detection of diverse HIV-1 genetic subtypes in the USA. Lancet 1995;346:1198-9.

7. Barin F, Courouce AM, Pillonel J, et al. Retrovirus study group of the French Society of Blood Transfusion: increasing diversity of HIV- $1_{M}$ serotype in French blood donors over a 10-year period (1985-1995). AIDS 1997;11:1503-8.

8. Zaidi I, Weinstock H, Parekh B, et al. Surveillance of HIV-1 subtypes in the United States, 1998-Present [abstract 215]. 7th Conference on Retroviruses and Opportunistic Infections, San Francisco, California, U.S.A., January 30-February 2, 2000.

9. Pieniazek D, Fonjungo P, Agwale S, et al. Frequency of new HIV variants among HIV-seropositive individuals world-wide [abstract 216]. 7th Conference on Retroviruses and Opportunistic Infections, San Francisco, California, U.S.A., January 30-February 2, 2000.

10. Alexander C, Dong W, Chan K, et al. HIV Non-B subtypes in a Large North American Cohort: Prevalence and Response to Antiretroviral Therapy [abstract 174]. 7th Conference on Retroviruses and Opportunistic Infections, San Francisco, California, U.S.A., January 30-February 2, 2000.

11. Dwyer DE, Herring BL, Wang B, et al. Multiple HIV-1 Subtypes Circulating in Australia. 7th Conference on Retroviruses and Opportunistic Infections[abstract 177]. 7th Conference on Retroviruses and Opportunistic Infections, San Francisco, California, U.S.A. January 30-February 2, 2000.

12. Lukashov VV, Huismans R, Bobkov AF, et al. Molecular Characterization of a Growing HIV-1 Epidemic among IDUs in Eastern Europe[abstract 178]. 7th Conference on Retroviruses and Opportunistic Infections, San Francisco, California, U.S.A., January 30-February 2, 2000

13. Pieniazek D, Yang C and Lal RB. 1998. Phylogenetic analysis of gp41 envelope of HIV-1 groups $\mathrm{M}, \mathrm{N}$, and $\mathrm{O}$ strains provides an 
alternate region for subtype determination. In The Human Retroviruses and AIDS 1998 Compendium. Los Alamos, NM: Los Alamos National Laboratory, 1998.

14. Hirsh MS, Brun-Vézinet F, D’Aquila RT, et al. Antiretroviral drug resistant testing in adult HIV-1 infection. JAMA 2000;283: 2417-26.

15. Salminen MO, Carr JC, Burke DS, et al. Identification of breakpoints in intergenotypic recombinants of HIV type 1 by bootscanning. AIDS Res Hum Retroviruses 1995;11:1423-5.

16. d'Arminio-Monforte A, Cozzi-Lepri A, Rezza G et al. Insights into the reasons for discontinuation of the first highly active antiretroviral therapy (HAART) regimen in a cohort of antiretroviral naive patients. AIDS 2000;14:499-507.
17. U.S. Centers for Disease Control and Prevention. 1993 Revised Classification System for HIV infection and expanded surveillance case Definition for AIDS among adolescents and adults. MMWR Morb Mortal Wkly Rep 1992;41:19-23.

18. Felsenstein J. 1996. PHYLIP: Phylogeny Inference Package, version 3.52. Seattle, WA: University of Washington, 1996.

19. Couturier E, Damond F, Roques P, et al. HIV-1 diversity in France, 1996-1998. AIDS 2000;14:289-96.

20. Wegner SA, Larder B, Hertogs K, et al. Use of a drug resistance sequence database to determine the prevalence of non-subtype $\mathrm{B}$ HIV-1 infections in US and European clinical cohorts [abstract 175]. 4th International Workshop on HIV Drug Resistance and Treatment Strategies. June 12-16, 2000, Sitges, Spain. 